\title{
Towards a Process for Negotiation of E-contracts Involving Web Services
}

\author{
Gabriel Costa Silva ${ }^{1}$, Itana Maria de Souza Gimenes ${ }^{2}$, Marcelo Fantinato ${ }^{3}$, Maria
Beatriz Felgar de Toledo ${ }^{4}$ \\ ${ }^{1}$ Coordenação do Curso de Ciências da Computação - Universidade Tecnológica \\ Federal do Paraná (UTFPR) \\ Caixa Postal 271 - 87301-006 - Campo Mourão - PR - Brasil \\ ${ }^{2}$ Departamento de Informática - Universidade Estadual de Maringá (UEM) - Maringá, \\ PR, Brasil \\ ${ }^{3}$ Curso de Sistemas de Informação - Escola de Artes, Ciências e \\ Humanidades/Universidade de São Paulo (EACH/USP) - São Paulo, SP - Brasil \\ ${ }^{4}$ Instituto de Computação - Universidade Estadual de Campinas (UNICAMP) - \\ Campinas, SP - Brasil \\ gabrielcosta@utfpr.edu.br, itana@din.uem.br, m.fantinato@usp.br, \\ beatrizeic.unicamp.br
}

\begin{abstract}
Organizations involved in cooperative business processes have different interests and points of view. A negotiation allows them to discuss their interests and requirements in order to reach an acceptable agreement. We propose an integrated web service negotiation process that considers human interaction and the use of different protocols. It focuses on the application of feature modelling to describe the negotiated services. Our contributions include: (i) the definition of a negotiation process; (ii) the definition of a conceptual model to support the negotiation of web services; (iii) reuse of artefacts generated throughout the negotiation process; and (iv) coverage of critical elements in the negotiation of electronic contracts.
\end{abstract}

\section{Introduction}

Organizations around the world are looking for means to ensure competiveness. Global cooperation has been considered a key factor. Business process outsourcing associated with internet technology offer a computational support that enable organizations to find partners, undertake negotiations and monitor the quality of contracted services even beyond geographical, cultural and technological limits [Grefen et al. 2006].

The web service technology, together with business process management concepts and tools offer a set of resources and standards [Papazoglou 2008; Weske 2010] that facilitate inter-organizational cooperation. Business processes (BP) are composed of electronic services (e-services). Service consumers look for e-service providers to compose optimal BP that join core internal competences and the outsourcing of e-services that are not their focus. These BP are regulated by electronic contracts (e-contracts).

A successful BP needs to take into account the different interests of organizations. Interests have to be discussed before an e-contract can be established. 
Thus, it is important to provide support for e-service negotiation [Grefen et al. 2006]. Negotiation is an interaction process amongst two or more partners in which their goal is to reach a mutually acceptable agreement [Zlatev 2002]. Electronic negotiation (enegotiation) is the process of conducting negotiations amongst business partners using electronic means. The partner in this process can be represented either by a human negotiator or a software agent [Rinderle and Benyoucef 2005]. The interactions amongst partners are guided by the rules of the negotiation protocols [Kim and Segev 2003]. These rules define how the partners exchange offerings and decide on how to proceed or end a negotiation. In the e-service context, the negotiation process is a requirement to reach an agreement [Grefen et al. 2006] and then establish an e-contract.

Several models [Lin 2008], frameworks [Comuzzi, Kritikos and Plebani 2009] and support systems [Kersten and Lai 2007] for e-negotiation have been proposed. There are also works related to negotiation protocols [Ueyama and Madeira 2001] and electronic markets [Mukhtar et al. 2009]. Although some studies regard the negotiation amongst software agents [Al-Aaidroos, Jailani and Mukhtar 2011], the negotiation literature shows that the current industry scenario is still based in human interaction [Lin 2008]. However, there is a lack of support to negotiation of e-services involving human interaction in the context of the web service technology. Thus, it is necessary to conceive and provide computational support to a process that guides the preparation and conduction of e-contract negotiation.

This paper presents a process that supports negotiation of e-contracts involving web services (WS-contracts). The process takes into account interaction amongst human negotiators and the use of different negotiation protocols. In addition, a feasibility study of the process is discussed. A prototype of a computer-aided support environment was developed for this purpose. The proposed negotiation process is provided within the context of a well-defined approach, called PL4BPM - Product Line for Business Process Management [Fantinato et al. 2009], and its respective support environment, called FeatureContract [Fantinato, Gimenes and Toledo 2010].

This paper is structured as follows. We present the research background, which is mainly concerned with BP, e-contract and PL4BPM. Next, the proposed WSnegotiation-process and its activities are presented. We then present a feasibility study developed to evaluate the proposed process, illustrated with snapshots of the developed prototype. Finally, we analyse related works and present the conclusions.

\section{Background}

Using web services technology, a software can be decomposed into self-contained, loosely coupled and language independent units [Papazouglou 2008]. BP can be used to compose web services; to integrate systems; to compose complex applications through services grouping and coordination; and, to establish partnerships in distributed system development. A BP consists of a set of tasks undertaken in a specific sequence to achieve a business goal [Weske 2010]. It also represents constraints on activities execution order as well as possible interactions between them.

An e-contract is a document used to represent an agreement amongst parties which is basically composed of: product or service definition; rights, obligations and prohibitions; and, actions to be taken in case of disagreements. Contracts can be complex and their establishment process is often cumbersome due to the large number 
of parameters involved in the selection of Quality of Service (QoS) attributes and levels. Thus, a negotiation amongst the parties is necessary to define the issues involved in establishing an e-contract [Grefen et al. 2006].

PL4BPM aims to provide support both to model variability in BP and web services, and to monitor WS-contracts throughout the process execution [Fantinato, Gimenes and Toledo 2010]. It is designed to model the artefacts involved in the negotiation amongst organizations willing to establish a WS-contract to regulate their cooperation. Feature modelling allows the representation of e-services and possible levels of QoS attributes. A feature model is represented through a tree-like diagram [Czarnecki, Helsen and Eisenecker 2005]. We have developed a feature meta-model in which the e-services feature diagram consists of two sub-trees, identified by the following pre-named root features: (i) e-services sub-tree that contains the features representing the e-services provided by an organization; and, (ii) qos-attribute sub-tree that contains the features representing the QoS attributes and levels which can be associated to the e-services.

\section{The WS-Negotiation process}

The WS-negotiation-process proposed in this paper has the following principles: (i) it focus on negotiation amongst organizations interested in undertaking a collaborative BP composed of web services; (ii) it promotes the reuse of the core artefacts produced throughout the negotiation processes; (iii) it supports decision making throughout the negotiation; (iv) it supports different negotiation protocols; and, (v) it takes into account interaction between human negotiators.

The WS-negotiation-process is carried out in the context of PL4BPM as presented in the previous section. Its conception was mainly supported by the negotiation framework proposed by Kim and Segev (2003) and the process model for enegotiation proposed by Kersten, Strecker and Law (2004). The process consists of eleven activities, as shown in Figure 1, divided into two life cycles: the Planning and Negotiation Agenda Settings and the Negotiating and Establishing WS-Contract. The first cycle defines the elements of the negotiation base, which are: (i) the negotiator roles; (ii) the business partners involved; (iii) target services of the negotiation; (iv) the negotiation variables for each service and their respective possible values; and, (v) the e-contract template. In the second cycle, the actual negotiation amongst the parties takes place by selecting the offered services, negotiating the variables of these services and finally, establishing the WS-contract.

\subsection{The Negotiation Conceptual Model}

The negotiation involves several related entities that compose the negotiation base. They are persistent elements defined in the first negotiation life cycle and used in the second life cycle to undertake the actual negotiation. Moreover, the negotiation base can be reused in further negotiations. The entities of the conceptual model are presented in Figure 2. This model is an important contribution of our work.

A Negotiation is conducted within a Negotiation Case, which is the main element of the WS-negotiation-process. A Negotiation Case belongs to only one Negotiator who is its owner. Each Negotiator has several Roles. An invited Negotiator assumes a Role within a Negotiation which defines its 
responsibility, such as "Credit Verification Service Provider". It is a specialization of the SOA roles, thus an organization can be a consumer or a provider, for example. The Role cardinality defines the number of negotiators, in that role, that can win the negotiation to provide and consume services at the end of the negotiation. For example, in a negotiation for tourist package, two airlines can win a trip, one to go and another to return, thus the cardinality is 2 (two). A Feature Model Template, associated with a Role, defines groups of e-services (e-service Group) which are related to Negotiation Variables. These variables represent gos Attribute(s) and e-service Property (ies).

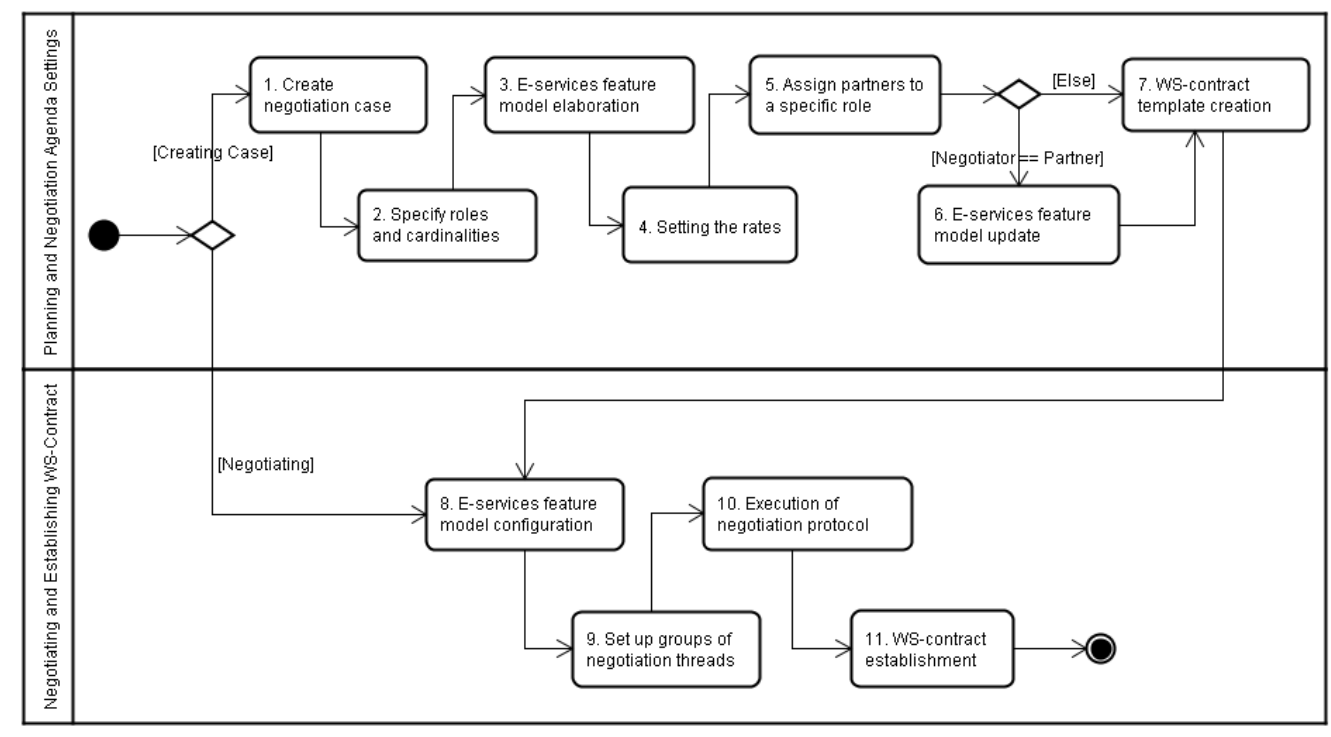

Figure 1. WS-negotiation-process model

A Negotiator is invited to participate of a Negotiation, playing a certain Role, based on a Profile which contains personal information. Each Negotiator receives a Feature Model Instance that can be updated according to the services that it can offer. According to the Negotiator interests, a Rating can be assigned to each e-service and Negotiation Variable. This Rating is part of the information base used by negotiators to define the negotiation strategy. The Negotiator (consumer) expresses its Interests by configuring the Feature Model Instance of its partner (provider).

The Negotiation is guided by a Protocol that defines a set of Activity(ies), like doing offers and counter-offers. Negotiation Thread(s) are formed of e-service Group(s) provided by a negotiation partner. A Negotiation Thread has a Rating. A Group of Thread has a Cardinality. A successful Negotiation produces one or more WS-contracts based on a WS-contract template.

\subsection{Activities of the Planning and Negotiation Agenda Settings life cycle}

The planning and negotiation agenda settings life cycle define the structure used through entire negotiation process, such as: items being negotiated (e-services and negotiation variable), partners and their roles, and rating for each negotiated issue. 
(1) Create negotiation case: this activity defines the objective of the negotiation and creates the negotiation case. The elements of a WS-contract negotiation are grouped within a negotiation case [Wu, Kersten and Benyoncef 2006]. The attributes of a negotiation case includes: a title, such as "Web-based graphical user interface", a brief description of the negotiation case that can be used in a directory where partners can find negotiations of their interest; keywords; privacy information of the case; and the deadline to end the negotiation in order to proceed to the WS-contract establishment.

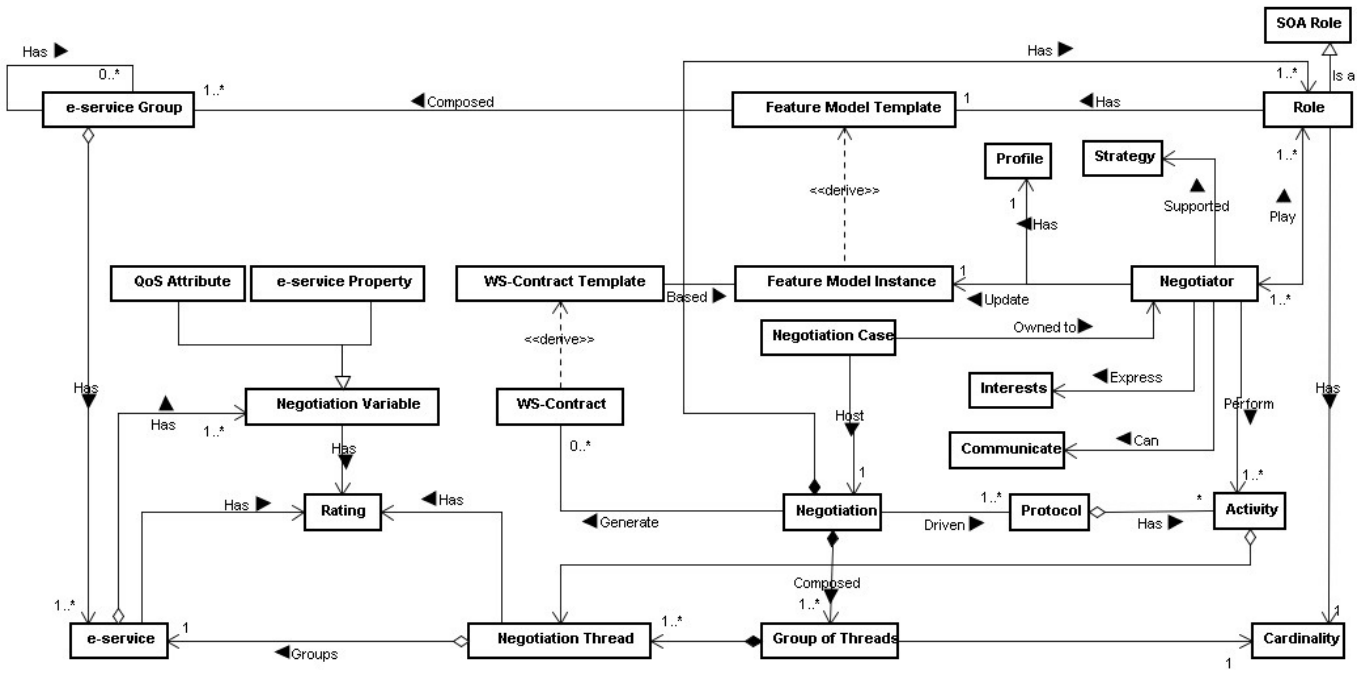

Figure 2. The conceptual model of negotiation

(2) Specify roles and cardinalities: this activity creates the roles that negotiators can have throughout a negotiation. The organization partners act within specific roles. A role groups a set of partners capable of providing the same type of service. In the same negotiation case there might be several roles. This activity also defines the negotiation direction, i.e. if the consumer search for providers or the providers search for consumers. Finally, cardinality has to be defined for each role. This allows the consumer organization to acquire services provided by different organizations.

(3) E-services feature model elaboration: e-services to be contracted are represented in the feature model as a template. This model is created and assigned to a role representing the set of services that the respective negotiator can offer. A negotiation starts with an instance of this model, associated with each negotiator, which is updated according to its capabilities to offer the services. They can have one or more associated negotiation variables, which represents an issue associated to the service that needs to be negotiated. It is an item of evaluation that supports the negotiator. An usual example of negotiation variable is price [Marchione et al. 2010]. PL4BPM considers two main types of negotiation variables: e-services properties and QoS attributes.

(4) Setting the rates: negotiators have different interests, whereas offering a service may be an advantage for a negotiator, it might not be for another. Thus, rates represent the importance of each negotiation item for a negotiator. They are assigned to: group of services, services and negotiation variables. The rates of a group of services vary from 0 (lowest) to 100 (highest). The rate of a service varies from 0 to the rate value of its group. After rating, we can see the importance of each service for the involved negotiators, such as organizations B, C and D. At this moment, only the negotiation 
driver (the one that creates the negotiation case) is capable of rating the items of its interest because the negotiators were not invited yet. When further negotiators start their participation, each of them has to assign rates to its feature model. These rates are strategic information because they directly express the negotiator preferences. Thus, they are private information that cannot be shared amongst negotiators. They are used to support the selection of the offers.

(5) Assign partners to a specific role: Negotiators are invited when they are previously known or they can be found in public directories. They can also offer themselves to participate of a negotiation when a call is made. When a negotiator is associated to a role, it receives the feature model instance based on feature model template for that role. It allows the negotiator to update its own feature model in the next process activity.

(6) E-services feature model update: a partner may either not be capable of providing the services or even provide additional services. Thus, it is necessary that the provider organization revise its feature model instance to represent its capabilities. In the negotiation literature, it is usually part of the negotiation agenda configuration [Kersten, Strecker and Law 2004]. It may demand modifications in the group of services, services and negotiation variables according to provider organizations.

(7) WS-contract template creation: this activity creates the WS-contract template that contains information that can be used in any similar contract established from the defined feature model.

\subsection{Activities of the Negotiating and establishing WS-contract life cycle}

The negotiating and establishing WS-contract life cycle uses the structure defined in the first life cycle to support the negotiation amongst partners. The services offered are selected to be contracted. The negotiation of the variable values of these services is carried out and, finally the WS-contract is established.

(8) E-services feature model configuration: the consumer selects the services and negotiation variables of its interest according to the instances of the feature model provided by the negotiators. There can be competing services and complementary ones.

(9) Set up groups of negotiation threads: each service negotiated between a provider and a consumer results in a negotiation thread. A group of negotiation thread has a cardinality that is inherited from a role and can be updated to define how many organizations can provide this group of services in particular.

(10) Execution of negotiation protocol: a negotiation protocol defines both style and rules that guide the bidding process. The negotiation proceeds or ends according to these rules. Our negotiation process can support the most common negotiation styles, such as bargain and auction. During the negotiation protocol execution, each service needs to be negotiated aiming at reaching an agreement amongst partners. Following the rules and observing the cardinalities, the winners of each negotiation thread are selected. The players can exchange short messages throughout our negotiation process. The amount of providers that can provide a group of services is defined by the cardinality of the role and the group of negotiation thread.

(11) WS-contract establishment: having defined the services and providers, the last activity can be executed. It consists of generating an instance of the WS-contract based 
on the template defined in first cycle of the negotiation process. The final WS-contract contains the service contracted by each winner organization of the negotiation.

\section{Feasibility Study}

A feasibility study was carried out to analyse the WS-negotiation process with the purpose of providing evidence of its feasibility and usage relevance. Thus, in this section we focus on the results obtained in this study. A computer-supported prototype was developed to automatize the proposed negotiation process. However, its details of operation will be discussed in other paper, thus they were omitted here. This prototype was used in the feasibility study to support the participants.

\subsection{The scenario domain}

The study was applied to a hypothetical scenario where an inter-organizational BP involves four types of organizations: a travel agency, hotels, airlines and car rental companies. This scenario involves: (i) organizations where one is a consumer organization (Travel Agency) and three are providers (Hotel, Airline and Car rental); (ii) negotiators, people that represent organizations throughout the negotiation; and (iii) services offered. From the WS-negotiation-process point of view, we consider that the travel agency is looking for providers to operate its BP, thus it will negotiate services to establish a WS-contract with selected providers. Feature models provide the specification of the services looked for the travel agency. An example of the feature model provided for the specification of the Airline services is shown in Figure 3. This feature model specifies the group of service Ticket management with services: Seeking ticket, Buying ticket; and Cancel ticket.

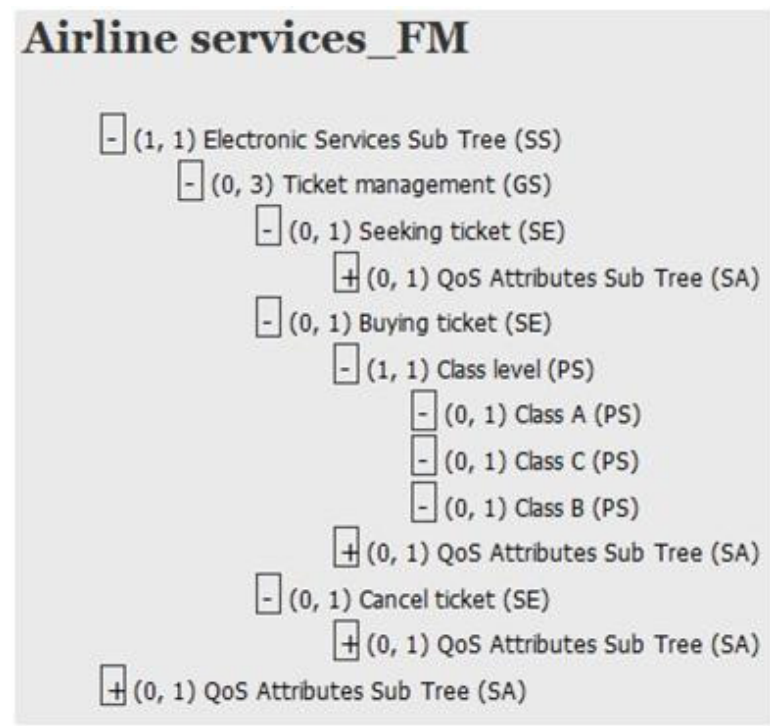

Figure 3. Feature model for the role of Airline Company

These services have a QoS Attributes sub-tree which is shown in Figure 4. It has two QoS Security and Availability. The possible levels for the Security are authentication by user (User Auth) or public authentication (Public auth). The QoS attribute Availability has the following control levels: $24 \times 7,24 \times 5$ and $8 \times 5$ (hours $x$ weekdays). 
In our hypothetical negotiation scenario the travel agency acts in the role of consumer interested in contracting services from the providers. Several organizations act in the role of providers with the following cardinalities:

- Hotel: cardinality 1 (one) - the travel agency covers only one destination so it is interested in contracting one hotel;

- Airline: cardinality 2 (two) - the agency can hire one company to go and another to return;

- Car rental: cardinality 999 (any number) - the client can rent different cars for each occasion.

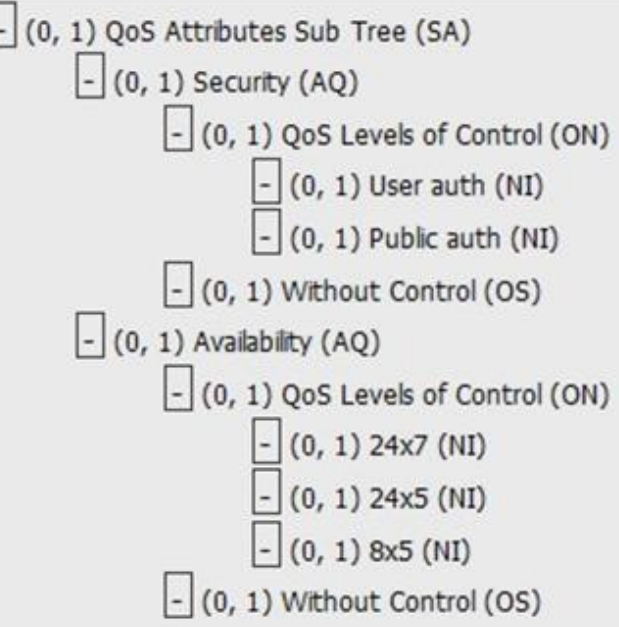

Figure 4. Feature model representing the QoS attributes of services

\subsection{Execution and discussion}

The feasibility study involved 5 (five) participants who are postgraduate students in computer science. The participants had three hours of training, two for the negotiation process techniques and one for the domain. They were divided into 4 (four) groups, one group with two participants and 3 (three) with one. Each participant was representing a role for one organization; except for the car rental organizations to which we had two participants. The travel agency was the negotiation driver. The negotiation protocols were bargain, auction and fixed-price. The participants were allocated in one room, each in a computer running the prototype of the WS-negotiation process support environment. The participants executed the eleven activities of the process. At the end they filled a questionnaire.

Data collected from the questionnaire were analysed regarding the feasibility and usage relevance of the WS-negotiation-process. Table 1 shows the analysed issues and the percentage of participant answers. Overall results show that participants were in favour of the WS-negotiation process feasibility and usage relevance. When asked about if the activity were clearly defined, $100 \%$ of the participants have agreed. Nevertheless, only $20 \%$ of the participants find the WS-negotiation-process easy to apply. 
They all considered the training satisfactory but $40 \%$ of them required assistance during the negotiation process. We did not consider this relevant, as the set of technological issues is really large for beginners.

Participants were asked if they consider the WS-negotiation process feasible. $100 \%$ of them considered the process useful and relevant so they would apply it again. They confirm that there are advantages of using the process as compared to informal negotiation. $100 \%$ of the participants agreed that all the partners involved can have benefits from the process support.

Table 1. Data collected from the participants of the feasibility study

\begin{tabular}{|c|c|c|}
\hline Question & Answer & $(\%)$ \\
\hline \multirow{2}{*}{$\begin{array}{l}\text { The WS-negotiation process training was } \\
\text { satisfactory }\end{array}$} & I agree, but I required assistance during the negotiation process & $40 \%$ \\
\hline & $\begin{array}{l}\text { I agree. I did not require any assistance during the negotiation } \\
\text { process }\end{array}$ & $60 \%$ \\
\hline The WS-negotiation activities are clearly defined & I agree & $100 \%$ \\
\hline \multirow{2}{*}{ The WS-negotiation process is easy to apply } & l agree & $20 \%$ \\
\hline & I disagree & $80 \%$ \\
\hline $\begin{array}{l}\text { The WS-negotiation process is feasible to all the } \\
\text { parts involved }\end{array}$ & I agree & $100 \%$ \\
\hline WS-negotiation process are feasible and useful & I agree. I would apply it again & $100 \%$ \\
\hline \multirow{2}{*}{$\begin{array}{l}\text { There are advantages of using the WS-negotiation } \\
\text { process as compared to informal negotiation }\end{array}$} & I agree, but it is hard to apply & $20 \%$ \\
\hline & I agree & $80 \%$ \\
\hline
\end{tabular}

We considered the prototype a threat of validity to the experiment, as it provides only the basic functions and a simple user interface. Some difficulties faced by the participants were related to the prototype stage of the support environment. A questionnaire was also applied to detect the experience of participants with the subject. Most of them have studied the subjects in the academic context but did not have previous contact with the involved concepts.

\section{Related Work}

Negotiation is a multidisciplinary area, thus it is discussed in [Bichler, Kersten and Strecker 2003]: psychology, economy and computer science. We could not find works that apply the same ideas of our approach towards modelling e-service negotiation. In particular, in the use of feature modelling to describe the negotiated services and its focus on artefact reuse. However, some works provided a solid background.

We mainly point out at: (i) the frameworks of Comuzzi, Kritikos and Plebani (2009), Mukhtar et al. (2009) and Kim and Segev (2003); (ii) the processes of Kersten, Strecker and Law (2004), Chiu et al. (2005) and Elfatraty and Layzell (2004); and, (iii) the model of Lin (2008) to support negotiation in electronic environment. Table 2 highlights items that we use to compare related works to ours. The items are: application area, multi-parties, multi-protocols, multi-items, multi-variables, support to decision making, human interaction throughout negotiation, e-contracts, web services and reuse of artefacts. 
Comuzzi, Kritikos and Plebani (2009) propose a framework that aims at discovering the negotiation protocol supported by the negotiators. It focuses on an important issue, which is the cooperation amongst partners, but it only deals with part of the negotiation process. Mukhtar et al. (2009) propose an integrated framework for electronic markets. Although it integrates technologies like internet and SMS to automate negotiation activities, it only deals with one scenario.

The Kim and Segev (2003) framework and the Kersten, Strecker and Law (2004) process provide basis for the conception of e-negotiation systems. They provide support for negotiation in dynamic environments; however, they do not take into account WS-contracts specific issues of web services, such as QoS.

Chiu et al. (2005) proposes a process and a metamodel for contract negotiation in B2B domain. However, it does not allow simultaneous negotiation of several items. Elfatatry and Layzell (2004) presents a negotiation process composed of three phases which defines favourite providers, roles and the establishment of a reliable negotiators database. However, details about the activities and artefacts are not provided.

Lin (2008) presents a conceptual model to specify a negotiation process in a service-oriented environment. The model defines a set of functionalities to each SOA element throughout the negotiation process as well as the interaction protocol amongst them. However, this model is limited to one provider and one consumer for each negotiation, which constrains the specification of more complex negotiation such as supply chains.

Table 2. Features of negotiation processes

\begin{tabular}{|c|c|c|c|c|c|c|c|}
\hline & Lin (2008) & Kim e Segev (2003) & $\begin{array}{c}\text { Comuzzi, } \\
\text { Kritikos e } \\
\text { Plebani } \\
(2009)\end{array}$ & $\begin{array}{c}\text { Mukhtar } \\
\text { et al. } \\
(2009)\end{array}$ & $\begin{array}{c}\text { Chiu et } \\
\text { al. } \\
(2005)\end{array}$ & $\begin{array}{c}\text { Kersten, } \\
\text { Strecker e } \\
\text { Law (2004) }\end{array}$ & $\begin{array}{c}\text { Elfatatry e } \\
\text { Layzell } \\
\text { (2004) }\end{array}$ \\
\hline Application area & $\begin{array}{c}\text { SOA } \\
\text { Negotiation }\end{array}$ & $\begin{array}{l}\text { Support to } \\
\text { negotiation on } \\
\text { dynamic }\end{array}$ & $\begin{array}{c}\text { Discovery of } \\
\text { available } \\
\text { protocols }\end{array}$ & $\begin{array}{c}\text { Electronic } \\
\text { market }\end{array}$ & $\begin{array}{l}\mathrm{B} 2 \mathrm{~B}, \\
\mathrm{~B} 2 \mathrm{C} \\
\mathrm{C} 2 \mathrm{C}\end{array}$ & \begin{tabular}{|c|} 
Electronic \\
Negotiation \\
Systems (ENS) \\
\end{tabular} & \begin{tabular}{|c|} 
Electronic \\
market of web \\
services \\
\end{tabular} \\
\hline Multi-parties & & $\mathrm{x}$ & $\mathrm{x}$ & $\mathrm{X}$ & $\mathrm{X}$ & $\mathrm{X}$ & \\
\hline Multi-protocols & & $\mathrm{x}$ & $\mathrm{x}$ & $\mathrm{X}$ & $\mathrm{x}$ & $\mathrm{X}$ & $\mathrm{X}$ \\
\hline \multicolumn{8}{|l|}{ Multi-items } \\
\hline Multi-variables & & $\mathrm{x}$ & $\mathrm{x}$ & $\mathrm{x}$ & $\mathrm{x}$ & $\mathrm{X}$ & \\
\hline $\begin{array}{c}\text { Support to decision } \\
\text { making }\end{array}$ & & $\mathrm{x}$ & & & & $\mathrm{x}$ & $\mathrm{x}$ \\
\hline $\begin{array}{c}\text { Human interaction } \\
\text { throughout negotiation }\end{array}$ & $\mathrm{x}$ & $\mathrm{x}$ & $\mathrm{x}$ & $\mathrm{X}$ & $\mathrm{x}$ & $\mathrm{X}$ & $\mathrm{x}$ \\
\hline Contracts & $\mathrm{x}$ & & & & $\mathrm{x}$ & & $\mathrm{X}$ \\
\hline Web services & $\mathrm{x}$ & & $\mathrm{x}$ & & & & $\mathrm{X}$ \\
\hline Reuse of artifacts & & & & & & $\mathrm{X}$ & $\mathrm{X}$ \\
\hline
\end{tabular}

Our negotiation process tackles the gaps of these works by taking into account web services negotiations which deals with: multi-parties, multi-protocols, multi-items, multi-variables, decision making, human interaction and artefact reuse.

\section{Conclusions}

This paper presents a negotiation process applied to support the establishment of WScontracts involving BP composed of web services. The negotiation process takes place 
in an environment that facilitates artefact reuse based on product line and feature modelling concepts. The negotiation process is composed of two main life cycles: (i) the Planning and Negotiation Agenda Settings and (ii) the Negotiating and Establishing WS-Contract. In addition to the proposed process a computer-supported prototype was developed and used in a feasibility study, which aimed at providing evidences of the feasibility and usage relevance of the process. The results confirmed that the proposed process is feasible and its usage is relevant for all the roles involved. However, we understand that an experiment with specialists is needed.

The contributions of this work include: (i) the definition of a negotiation process; (ii) the definition of a conceptual model to support the negotiation of eservices; (iii) reuse of artefacts generated throughout the negotiation process; and (iv) coverage of critical elements in the negotiation of electronic contracts, such as role, features of electronic services and contract models.

Future work includes the study of renegotiation in the context of the proposed conceptual model of negotiation. Although renegotiation is similar to negotiation in terms of roles, protocols, strategies and others, it appears more rarely in the literature. [Vecchiato et al. 2010] extended the WS-contract feature meta-model to contain actions and restrictions to contemplate renegotiation. Therefore, this new WS-Contract model will be further incorporated to our proposed negotiation conceptual model of negotiation to deal with renegotiation.

\section{References}

Al-Aaidroos, M., Jailani, N., Mukhtar, M. (2011) "Agent-based negotiation framework for web service's SLA", Proceedings of the 7th International Conference on Information Technology in Asia: Emerging Convergences and Singularity of Forms (CITA 2011), Kuching, pp. 1-7.

Bichler, M., Kersten, G. and Strecker, S. (2003). Towards a Structured Design of Electronic Negotiations. In Group Decision and Negotiation, Vol. 12, No. 1, pp. 311335.

Comuzzi, M., Kritikos, K. and Plebani, P. (2009) "A semantic-based framework for supporting negotiation in Service Oriented Architectures", Proceedings of the 2009 IEEE Conference on Commerce and Enterprise Computing (CEC 2009), London, pp. 137-145.

Chiu, D.K., Cheung, S.C., Hung, P.C., Chiu, S.Y. and Chung, A.K. (2005). Developing e-Negotiation support with a meta-modeling approach in a web services environment. In Decision Support Systems, Vol. 40, No. 1, pp. 51-69.

Czarnecki, K., Helsen, S. and Eisenecker, U. (2005). Staged Configuration through Specialization and Multi-Level Configuration of Feature Models. In Software Process: Improvement and Practice, Vol. 10, No. 2, pp. 143-169.

Elfatatry, A. and Layzell, P. (2004). Negotiating in service-oriented environments. In Communications of the ACM, Vol. 47, No. 8, pp. 103-108.

Fantinato, M., Gimenes, I.M.S. and Toledo, M.B.F. (2009) "Product Line in the Business Process Management Domain", in K.C. Kang, V. Sugumaran and S. Park (eds.), Applied Software Product Line Engineering, Auerbach Publications, London, pp. 497-530. 
Grefen, P., Ludwig, H., Dan, A. and Angelov, S. (2006). An analysis of web services support for dynamic business process outsourcing. In Information and Software Technology, Vol. 48, No. 11, pp. 1115-1134.

Kersten, G.E., Strecker, S.E. and Law, K.P. (2004) "Protocols for electronic negotiation systems: theoretical foundations and design issues", in K. Bauknecht, M. Bichler and B. Pröll (eds.), EC-Web 2004 LNCS Vol. 3182, Springer, Berlin, pp. 106-115.

Kersten, G.E. and Lai, H. (2007). Negotiation support and e-negotiation systems: An overview. In Group Decision and Negotiation, Vol. 16, No. 6, pp. 553-586.

Kim, J.B. and Segev, A. (2003) "A Framework for Dynamic eBusiness Negotiation Processes", Proceedings of the IEEE International Conference on E-Commerce Technology, Los Alamitos, pp. 84-91.

Lin, J.A. (2008). A conceptual model for negotiating in service-oriented environments. In Information Processing Letters, Vol. 108, No. 4, pp. 192-203.

Marchione, F., Fantinato, M., Toledo, M.B.F. and Gimenes, I.M.S. (2010). "Price Definition in the Establishment of Electronic Contracts for Web Services". In International Journal of Web and Grid Services (IJWGS), to be published: Vol. 6, No. 4, pp. 357-384.

Mukhtar, M., Yahya, Y., Jailani, N., Abdullah, S. and Abdullah, Z. (2009) "An integrated e-marketplace framework: A case study of wood-based products industry in Malaysia", Proceedings of the 2009 International Conference on Electrical Engineering and Informatics (ICEEI 2009), Bangi, pp. 314-320.

Papazoglou, M. (2008) Web Services: Principles and Technology, Prentice Hall, Harlow.

Rinderle, S. and Benyoucef, M. (2005) "Towards the automation of e-negotiation processes based on Web services - A modeling approach", in A.H.H. Ngu, M. Kitsuregawa, E.J. Neuhold, J. Chung and Q.Z. Sheng (eds), WISE 2005 LNCS Vol. 3806, Springer, Berlin, pp. 443-453.

Ueyama, J. and Madeira, E.R.M. (2001) "An Automated Negotiation Model for Electronic Commerce", Proceedings of the Fifth International Symposium on Autonomous Decentralized Systems Autonomous Decentralized Systems, Dallas, pp. 29-36.

Zlatev, Z.V. (2002) "Examination of the negotiation domain", Technical Report, n.0239, Centre for Telematics and Information Technology, University of Twente, Enschede.

Vecchiato, D.A., Toledo, M.B.F., Fantinato, M. and Gimenes, I.M.S. (2010) "A Feature-based Toolkit for Electronic Contract Negotiation and Renegotiation", Proceedings of the IADIS International Conference WWW/Internet 2010 (IADIS ICWI), Timisoara, pp. 3-10.

Weske, M. (2010) Business Process Management: Concepts, Languages, Architectures, Springer, Berlin.

Wu, S., Kersten, G.E. and Benyoncef, M. (2006) "INSS-a new approach in designing Web-based negotiation support systems", Proceedings of the Montreal Conference on e-Technologies, Montreal, pp. 1-15. 